\title{
A stented bovine pericardial prosthesis in the pulmonary position
}

Hanna Pragt, MSc, ${ }^{a}$ Mirthe H. Schoots, MD, ${ }^{\mathrm{b}}$ Ryan E. Accord, MD, ${ }^{\mathrm{a}}$ Sara C. Arrigoni, MD, ${ }^{\mathrm{a}}$

Rolf M. Berger, MD, PhD, ${ }^{\mathrm{c}}$ Massimo A. Mariani, MD, PhD, ${ }^{a}$ Tineke P. Willems, MD, PhD, ${ }^{d}$

Tjark Ebels, $\mathrm{MD}, \mathrm{PhD},{ }^{\mathrm{a}, \mathrm{e}}$ and Joost P. Van Melle, $\mathrm{MD}, \mathrm{PhD}^{\mathrm{f}}$

\section{ABSTRACT}

Background: Pulmonary valve replacement is very common among patients with congenital heart disease. The Carpentier Edwards Perimount valve (Edwards Lifesciences, Irvine, Calif), which was originally designed for the aortic position is among the most implanted valves. We aim to describe the follow-up of this valve in the pulmonary position.

Methods: Patients with a Perimount valve implanted between 2003 and 2013 in the University Medical Center Groningen were followed for the primary end point reintervention, defined as surgical or transcatheter valve replacement. Secondary end point was the occurrence of valve failure, defined as significant valvular regurgitation or stenosis. Explanted valves were histologically examined.

Results: Forty-five patients (median age at operation 27.8 years, $55.6 \%$ women) had a mean follow-up duration of $5.8 \pm 3.3$ years. There were 7 reinterventions ( 5 surgical and 2 transcatheter). Freedom from reintervention was respectively $95 \% \pm 4 \%$ and $83 \% \pm 8 \%$ at 5 - and 10 - years of follow-up. Freedom from valve failure was $75 \% \pm 4 \%$ at 2 years, $65 \% \pm 8 \%$ at 5 years of follow-up and $57 \% \pm 10 \%$ at 10 years of follow-up. Morphology evaluation $(n=4)$ showed stiffened valves in the open position, with extensive fibrous tissue overgrowth on the leaflets and a variable proliferation of myofibroblasts.

Conclusions: The Perimount valve has adequate function in the pulmonary valve position at 5 years of follow-up, although after 10 years of follow-up valve failure and reinterventions are common. Explanted valves show retraction and stiffening of the leaflets due to a fibrotic layer on both sides of the leaflet. (J Thorac Cardiovasc Surg 2020;159:1063-71)

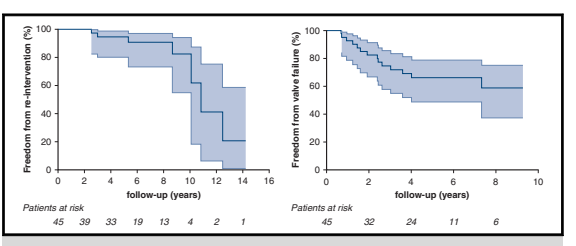

Cumulative freedom from reintervention and valve failure, including $95 \%$ confidence interval.

\section{Central Message}

The Carpentier Edwards Perimount valve (Edwards Lifesciences, Irvine, Calif) has adequate function in the pulmonary valve position at 5 years' follow-up, yet after 10 years valve failure is common. Explanted valves show retraction of the leaflets.

\section{Perspective}

This study contributes to the knowledge on prosthetic heart valves in the pulmonary position. We have shown that the Carpentier Edwards Perimount valve (Edwards Lifesciences, Irvine, Calif) has adequate function in the pulmonary valve position; however, after 10 years valve failure and reinterventions are common. More research is necessary in the field of prosthetic valves for this position to facilitate validated clinical valve choices.

See Commentaries on pages 1072 and 1074.
A wide spectrum of congenital heart diseases require pulmonary valve replacement (PVR) with or without reconstruction of the right ventricular outflow tract. There are

\footnotetext{
From the Departments of ${ }^{\mathrm{a}}$ Cardiothoracic Surgery, ${ }^{\mathrm{c}}$ Pediatric Cardiology, ${ }^{\mathrm{d}}$ Radiology, and ${ }^{\mathrm{f}}$ Cardiology, and ${ }^{\mathrm{b}}$ Pathology Section, Department of Pathology and Medical Biology, University Medical Center Groningen, University of Groningen, Groningen, The Netherlands; and ${ }^{\mathrm{e}}$ Department of Cardiothoracic Surgery, Academic Medical Centre, University of Amsterdam, Amsterdam, The Netherlands.

Received for publication Aug 10, 2018; revisions received March 31, 2019; accepted for publication May 20, 2019; available ahead of print Aug 7, 2019.

Address for reprints: Joost P. van Melle, MD, PhD, Department of Cardiology, University Medical Center Groningen, University of Groningen, Hanzeplein 1, 9700RB Groningen, The Netherlands (E-mail: j.p.van.melle@umcg.nl). $0022-5223 / \$ 36.00$

Copyright (c) 2019 by The American Association for Thoracic Surgery https://doi.org/10.1016/j.jtcvs.2019.05.086
}

several options for PVR, including homografts, bovine jugular vein conduits, mechanical prosthetic valves, and biological prosthetic valves. Homograft and bovine jugular vein conduits have limited availability and sizes, while mechanical valves are associated with higher rates of valve thrombosis requiring lifelong use of anticoagulation medication. ${ }^{1}$ As a result, biological aortic valve prostheses are widely used for PVR. When the occasion rises, the stented

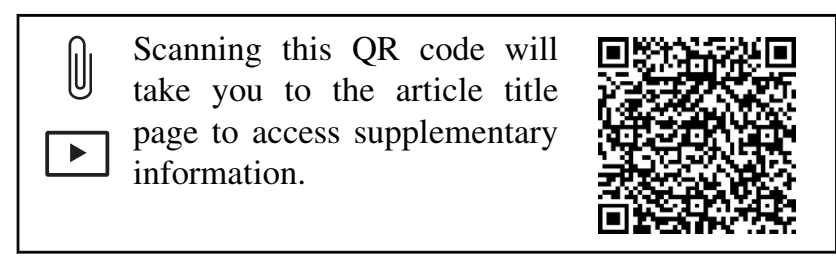




\section{Abbreviations and Acronyms}

LVEF $=$ left ventricular ejection fraction

PPEC = percentage of predicted exercise capacity

PVR = pulmonary valve replacement

RVEDD $=$ right ventricular end diastolic diameter

TAPSE $=$ tricuspid annular plane systolic excursion

TOF $=$ tetralogy of Fallot

bovine pericardial Carpentier Edwards Perimount valve (Edwards Lifesciences, Irvine, Calif) is among the most implanted bioprosthetic valves in the pulmonary position. Originally designed for left-sided valvular heart disease, the Perimount valve has shown excellent results in the aortic and mitral valve position. ${ }^{2-4}$ However, data on its durability and functionality in the pulmonary valve position are scarce. The Perimount valve has shown mixed results in the pulmonary position so far. ${ }^{5-12} \mathrm{~A}$ large study by Nomoto and colleagues ${ }^{12}$ showed very good results in terms of reintervention for the Perimount valve compared with the Mitroflow valve (Sorin Group USA Inc, Arvada, Colo), whereas the studies by Jang and colleagues ${ }^{7,11}$ suggested higher rates of valve failure for the Perimount valve compared with porcine valves. Of note, both studies were performed in a young population with a relative short follow-up duration. Most studies are limited in echocardiographic valve evaluation and others include heterogeneous populations in terms of implanted valve type and age.

More uniform long-term research on the functionality of prosthetic valves in the pulmonary position is essential to provide patients and physicians with a well-founded choice, when selecting a prosthetic valve for this position. In this retrospective observational study, we evaluate the Perimount valve in an adolescent/adult population. Both re-intervention rates as well as valve function (based on echocardiograph images) are evaluated, giving a complete representation of valve functioning.

\section{METHODS \\ Patients}

All patients in whom a Perimount aortic valvular prosthesis was implanted in the pulmonary position between 2003 and 2013 in the University Medical Center Groningen were included in this study. The Perimount series include the Perimount, Perimount Magna, and the Perimount Magna Ease valve. The valves consist out of bovine pericardial leaflets mounted on a flexible cobalt-chromium alloy stent. Valve choice was based on surgeon's preference and patient's wishes. Valve replacement was performed through a median sternotomy with the use of cardiopulmonary bypass. Indication for PVR was based on a combination of criteria, including but not limited to, symptoms related to pulmonary regurgitation, QRS complex duration, right ventricular volume, right ventricular ejection fraction, maximum oxygen capacity uptake, left ventricular volume, ventricular tachycardia and tricuspid regurgitation. Data were retrieved retrospectively using electronically stored patient files up to October 2017. No identifiers were linked to patients in this database. Applicable legislation in the Netherlands stipulates that retrospective anonymized chart research is not subjected to institutional review board review.

\section{End Points}

The primary end point was reintervention, defined as surgical or transcatheter (valve-in-valve) replacement of the prosthetic heart valve. Secondary end point was the occurrence of prosthetic valve failure, which was defined as the occurrence of at least moderate regurgitation or moderate stenosis. The other secondary end point was endocarditis.

\section{Echocardiography}

Valve functioning was determined by standard clinical 2-dimensional transthoracic Doppler echocardiography performed by trained specialists as part of routine clinical care. The following parameters were evaluated with color and Doppler echocardiography: prosthetic valve regurgitation and stenosis, tricuspid valve regurgitation, all of which were graded according to European Association of Echocardiography/American Society of Echocardiography and European Association of Cardiovascular Imaging guidelines. ${ }^{13-15}$ Additionally, left ventricular function measured as left ventricular ejection fraction (LVEF), right ventricular function measured as tricuspid annular plane systolic excursion (TAPSE) and right ventricular end diastolic diameter (RVEDD) in the 4-chamber apical view were determined. Regurgitation and stenosis were evaluated postoperatively in the aftermath of a recent implantation and during regular outpatient clinic visit. Additional parameters were evaluated postoperatively and during the last outpatient clinic visit.

\section{Exercise Capacity}

Exercise capacity was evaluated using the percentage of predicted exercise capacity (PPEC), calculated by dividing the achieved exercise level by the predicted exercise level. Exercise capacity was evaluated as part of routine clinical care. Testing was performed using a bicycle ergometer or treadmill. Routine clinical care protocols were followed: the Bruce, modified Bruce, 10 Watt, or 15 Watt programs. Patients were permanently monitored with echocardiogram, blood pressure, and oxygen saturation during the test. Exercise levels were evaluated using either maximum oxygen capacity or the maximum workload. Predicted workload was calculated as described by Ascoop and colleagues, ${ }^{16}$ and predicted maximum oxygen capacity was determined with the Wasserman/Hansen equation. ${ }^{17}$

\section{Pathologic Evaluation}

Of the surgically explanted valves $(\mathrm{N}=5), 4$ valves were histologically examined and compared with a nonimplanted Perimount valve. Specimens were described macroscopically, and samples were formalin fixed and paraffin embedded according to standard operating procedures in our routine diagnostic pathology laboratory. Series of sections were cut at $4 \mu \mathrm{m}$ thickness and mounted onto glass slides. We performed hematoxylin and eosin stain, Verhoeff-van Gieson stain, Alcian Blue stain, Van Kossa stain, and Martius scarlet blue stain using standard staining protocols. Besides histomorphology, slides were stained with different immunohistochemistry markers to differentiate between cell types. Alpha smooth muscle actin staining (clone 1A4; Agilent Technologies, Santa Clare, Calif) and Caldesmon (clone h-CD; Agilent Technologies) were performed to stain myofibroblasts. CD45 (clone 2B11+PD7/26; Roche Holding Ltd, Basel, Switzerland) was used to identify inflammatory cells. To identify endothelium, CD31 (clone JC40; Roche Holding Ltd) was applied.

\section{Statistical Analysis}

Normally distributed continues variables are presented as mean \pm standard deviation. Not normally distributed continues variables 
are presented as medians and ranges. Dichotomous variables are presented as numbers and percentages. Paired samples Student $t$ test was used to compare LVEF, TAPSE, RVEDD, and PPEC postoperatively and at last visitation. In case of reintervention, the last visit before reintervention was considered for evaluating echocardiography parameters. Kaplan-Meier analyses and life tables were used to evaluate time to event. Cox proportional hazard methodology was used to determine relationships between the covariates and time to event. Age, gender, tetralogy of Fallot (TOF), number of sternotomies, labeled size, TAPSE, LVEF, PPEC, and RVEDD were evaluated as univariables, and all variables with a $P$ value $<.1$ were include in a multivariable model. Additionally, a multivariate model including age and gender was created. All statistical analyses were performed with IBM SPSS Statistics version 23 (IBM-SPSS Inc, Armonk, NY).

\section{RESULTS}

We included a total of 45 patients who received a Perimount valve in the pulmonary position; this was $21.2 \%$ out of a total of 212 PVR patients between 2003 and 2013. Of these 212 valves, 98 patients received a Contegra valve (Medtronic, Minneapolis, Minn) (46\%) of which 9 were older than age 16 years at implantation, 47 received a mechanical valve $(22 \%), 13$ received a Melody valve (Medtronic) $(6.1 \%), 5$ received a porcine valve $(2.3 \%)$, and 4 received a homograft $(1.9 \%)$.

\section{Baseline Characteristics}

As depicted in Table 1, median age at time of valve implantation was 27.8 years (range, 15.1-59.2 years). There were 3 patients younger than age 18 years. The study sample comprised $55.6 \%$ women. The main underlying cardiac diagnosis was TOF $(68.9 \%)$, followed by congenital pulmonary regurgitation and/or stenosis $(24.4 \%)$. For the patients with TOF, median time between total correction and implantation of the Perimount valve was 25.5 years (interquartile range, 20.0-32.8 years). Of the patients included, $98 \%$ had at least 1 prior sternotomy. Median implanted valve size was $25 \mathrm{~mm}$ (range, $23-29 \mathrm{~mm}$ ), 22 (48.9\%) of patients received a $25-\mathrm{mm}$ valve.

\section{Reintervention}

Mean follow-up duration was $5.8 \pm 3.3$ years; mean age at follow-up was $39.1 \pm 12.2$ years. Two patients died during the follow-up period. One patient died in the early aftermath 3 days postsurgery related to postoperative cerebral ischemia. The other fatality was 3 years after PVR due to end-stage right ventricular heart failure, with a well-functioning valve. In total there were 7 reinterventions of which were 2 transcatheter (valve-in-valve) interventions and 5 were surgical reinterventions. Cumulative freedom from reintervention was $95 \% \pm 4 \%$ at 5 years, $83 \% \pm 8 \%$ at 10 years, and $12 \% \pm 16 \%$ at 15 years postimplantation as shown in Figure 1. For 6 patients, severe valve regurgitation was the main reason for reintervention and for the remaining patient endocarditis was the cause for reoperation.
TABLE 1. Baseline characteristics

\begin{tabular}{|c|c|}
\hline Characteristic & Result \\
\hline Female gender & $25(55.6 \%)$ \\
\hline Number of thoracotomies & $1 \pm 1.0$ \\
\hline 0 & $1(2.2 \%)$ \\
\hline 1 & $29(64.4 \%)$ \\
\hline 2 & $9(20.0 \%)$ \\
\hline 3 & $3(6.7 \%)$ \\
\hline 4 & $2(4.4 \%)$ \\
\hline 5 & $1(2.2 \%)$ \\
\hline \multicolumn{2}{|l|}{ Principal cardiac diagnosis } \\
\hline Tetralogy of Fallot & $31(68.9 \%)$ \\
\hline Pulmonary stenosis & $11(24.4 \%)$ \\
\hline Truncus arteriosus & $1(2.2 \%)$ \\
\hline Pulmonary atresia & $1(2.2 \%)$ \\
\hline Aortic valve replacement & $1(2.2 \%)$ \\
\hline \multicolumn{2}{|l|}{ Previous procedures } \\
\hline Aortic pulmonary shunt & $10(22.2 \%)$ \\
\hline Total correction & $31(68.9 \%)$ \\
\hline Pulmonary valvulotomy & $13(28.9 \%)$ \\
\hline VSD closure & $4(8.9 \%)$ \\
\hline Pulmonary valve replacement & $7(15.6 \%)$ \\
\hline Other & $8(17.8 \%)$ \\
\hline History of endocarditis & $2(4.4 \%)$ \\
\hline Valve size (mm) & $25(23-29)$ \\
\hline Age at implantation (y) & $31.26 \pm 12.54^{*}$ \\
\hline \multicolumn{2}{|l|}{ Concomitted procedures } \\
\hline RVOT correction & $6(13.3 \%)$ \\
\hline VSD closure & $1(2.2 \%)$ \\
\hline Tricuspid valve repair & $4(8.9 \%)$ \\
\hline Other & $3(6.7 \%)$ \\
\hline \multicolumn{2}{|l|}{ Echocardiographic variables } \\
\hline Tricuspid regurgitation & $15(33.3 \%)$ \\
\hline $\operatorname{LVEF}(\%)$ & $51.6 \pm 5.0$ \\
\hline TAPSE $(\mathrm{mm})$ & $14.3 \pm 4.0$ \\
\hline $\operatorname{RVEDD}\left(\mathrm{mm} / \mathrm{m}^{2}\right)$ & $41.9 \pm 6.9$ \\
\hline PPEC $(\%)$ & $67.4 \pm 16.8$ \\
\hline
\end{tabular}

Values are presented as mean \pm standard deviation, $\mathrm{n}(\%)$, or median (range). VSD, Ventricular septal defect; $R V O T$, right ventricular outflow tract; $L V E F$, left ventricular ejection fraction; TAPSE, tricuspid annular plane systolic excursion; RVEDD, right ventricular end diastolic diameter; $P P E C$, predicted percentage exercise capacity. *Range, 15.1 to 59.2 years.

\section{Secondary End Points}

Freedom from valve failure (at least moderate regurgitation or moderate stenosis) was $75 \% \pm 4 \%$ at 2 years of follow-up, $65 \% \pm 8 \%$ at 5 years of follow-up and $57 \% \pm 10 \%$ at 10 years of follow-up. At final follow-up, 10 patients had severe regurgitation $(22.2 \%), 3$ patients had moderate regurgitation $(6.7 \%), 9$ patients had moderate stenosis $(20.0 \%)$, and 1 patient had severe stenosis $(2.2 \%)$. There was 1 patient with pulmonary valve stenosis without valvular regurgitation. In total, there were 5 cases of endocarditis $(11.1 \%$ of the total study cohort). Cumulative 


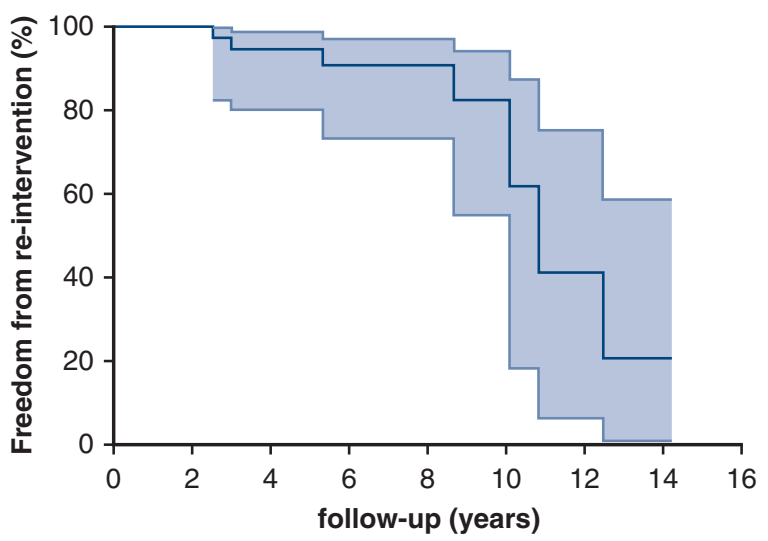

Patients at risk

$\begin{array}{llllllll}45 & 39 & 33 & 19 & 13 & 4 & 2 & 1\end{array}$

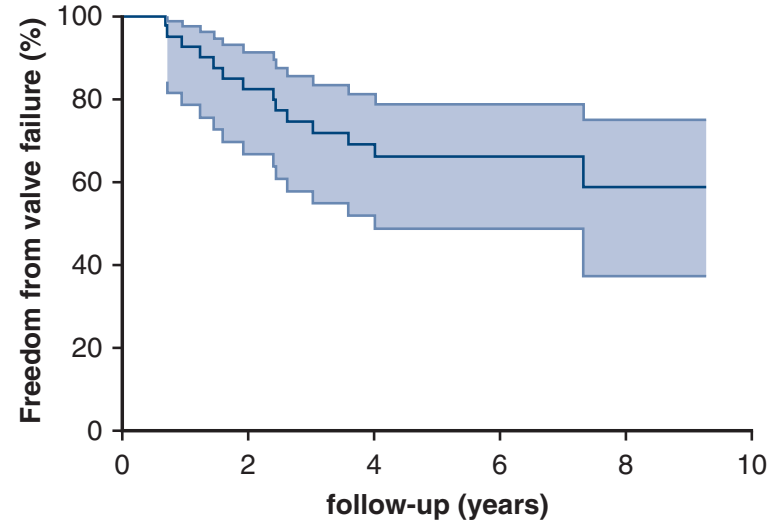

Patients at risk

$\begin{array}{lllll}45 & 32 & 24 & 11 & 6\end{array}$

FIGURE 1. Cumulative freedom from reintervention and freedom from valve failure during 15 -year follow-up of Carpentier Edwards Perimount valves (Edwards Lifesciences, Irvine, Calif) in the pulmonary position, including the $95 \%$ confidence interval. Reintervention was defined as surgical or transcatheter valve replacement. Valve failure was defined as moderate or severe valve regurgitation or stenosis.

freedom from endocarditis was $89 \% \pm 5 \%$ at 5 years postPVR and $83 \% \pm 8 \%$ at 10 years post-PVR (Figure 2). Mean time to develop endocarditis was $3.03 \pm 1.9$ years (range, 0.89-5.22 years) after PVR.

Right ventricular function measured by TAPSE improved from $14.3 \pm 4.0 \mathrm{~mm}$ postoperatively to $16.9 \pm 3.8 \mathrm{~mm}$ at final visit $(P<.001)$, although the RVEDD increased from $41.9 \pm 6.9 \mathrm{~mm} / \mathrm{m}^{2}$ postoperatively to $44.03 \pm 6.0 \mathrm{~mm} / \mathrm{m}^{2}$ at last visit $(P=.001)$. Postoperative left ventricular function was stable during follow up (LVEF $51.6 \% \pm 5.0 \%$ compared with $51.5 \% \pm 6.7 \%$ at last visit) $(P=.27)$. Exercise capacity measured by PPEC was increased from $67.4 \% \pm 16.8 \%$ at first test postoperatively to $72 \% \pm 15.7 \%$ at final visit $(P=.05)$.

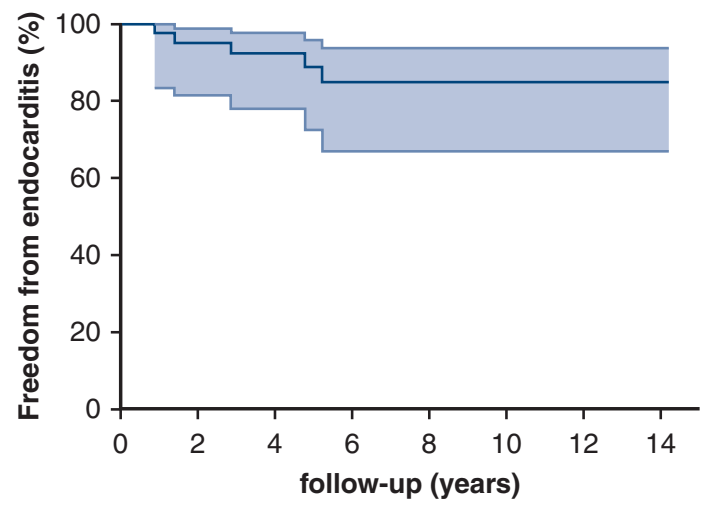

patients at risk

$$
\begin{array}{llllllll}
45 & 37 & 31 & 17 & 12 & 3 & 2 & 1
\end{array}
$$

FIGURE 2. Cumulative freedom from endocarditis during a 10-year follow-up of Carpentier Edwards Perimount valves (Edwards Lifesciences, Irvine, Calif) in the pulmonary position, including $95 \%$ confidence interval. Endocarditis was defined as clinically treated endocarditis.
Univariate Cox proportional hazard analyses showed no effect on time to valve failure for age, gender, underlying disease (divided by TOF vs other), use of anticoagulation, RVEDD, TAPSE, LVEDD, and valve size. For time to reoperation RVEDD was both univariable and in a multivariable model with age and gender, associated with reoperation (hazard ratio, 1.39; 1.04-1.85; $P=.027$ ). There were no other significant associations for any of these variables (Table E1).

\section{Morphologic Findings}

Among the explanted valves $(\mathrm{N}=5) 1$ valve was explanted due to active endocarditis and 1 patient was treated for suspected endocarditis years before the operation. The histologically examined valves $(n=4)$ showed severe malfunctioning due to thickening and valve retraction as shown in Figures 3 and 4. Histologically, all valves showed central collagenous thickening, with fibrosis on the surface on both sides of the leaflet (Table 2). This fibrosis showed variable proliferation of myofibroblasts (Figure 4). No valves showed an endothelial lining. One valve showed central dystrophic calcification. In addition, some valves showed fibrin deposition/thrombosis at the surface. Three out of the 4 valves showed inflammatory cells. Our findings were compared with a nonimplanted Perimount valve, which showed no inflammation, collagenous thickening, fibrosis, or endothelial lining at all (Figure 5).

\section{DISCUSSION}

Bioprosthetic aortic valves are used off-label for replacement of pulmonary valves. For this purpose, the Perimount valve is among the most widely used stented bioprosthetic valves. There is a scarcity of data on the long-term functionality of these valves in the pulmonary position. The results 


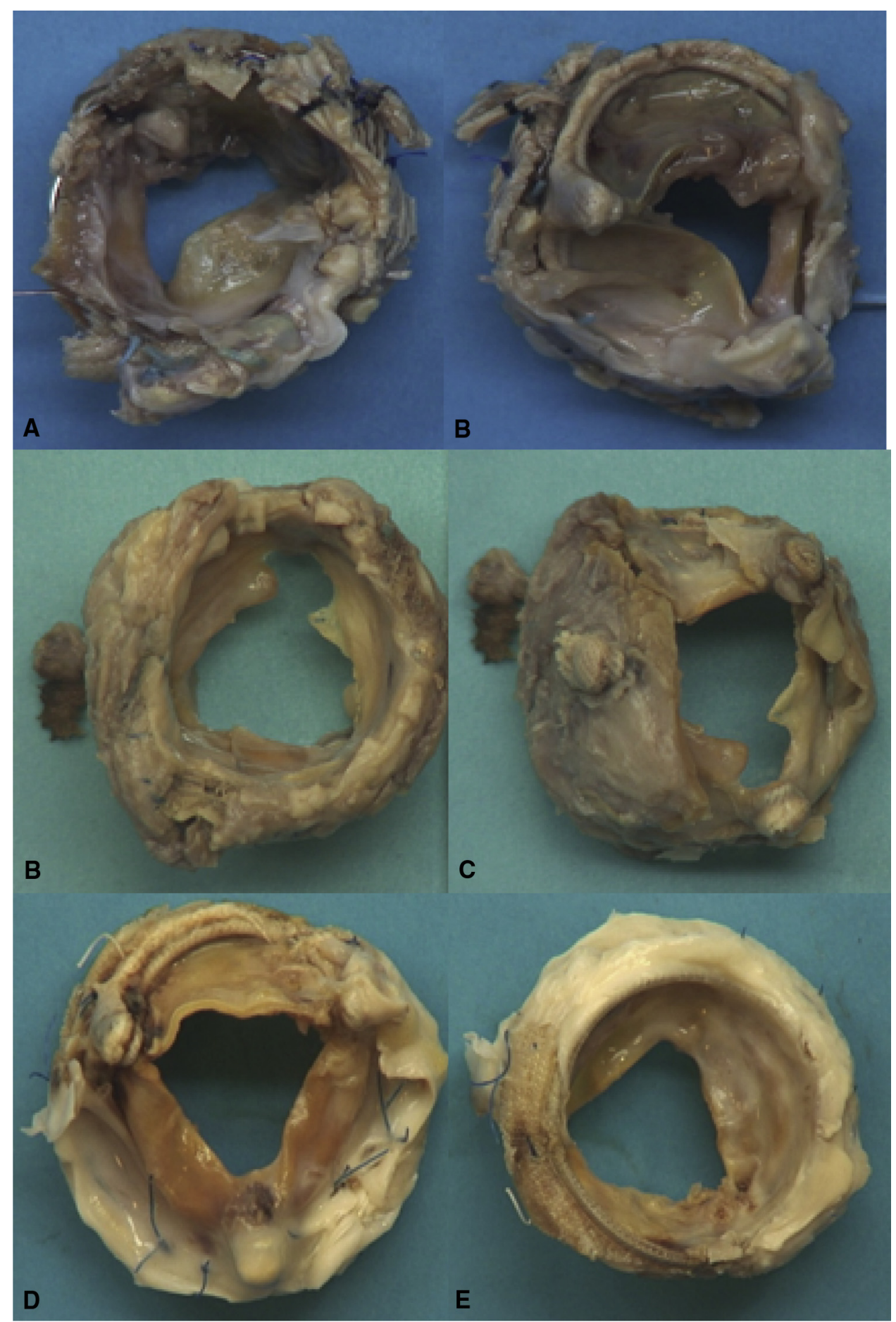

FIGURE 3. Views of 3 explanted bovine pericardial Carpentier Edwards Perimount valves (Edwards Lifesciences, Irvine, Calif) with retracted leaflets. A and B, Front and back view of valve with an implanted duration of 5 years. C and D, Front and back view of valve with an implanted duration of 10 years. D and E, Front and back view of valve with an implanted duration of 13 years.

of this study show that short-term freedom from reintervention has been acceptable for the Perimount valve in the pulmonary position. Although numbers are limited after 10-year follow-up, prosthetic failure and reinterventions were common. There was no mortality specifically related to valve failure. All surgically explanted and examined valves showed the same features: Valve leaflets were degenerated and stiffened and complete closure was impossible. 


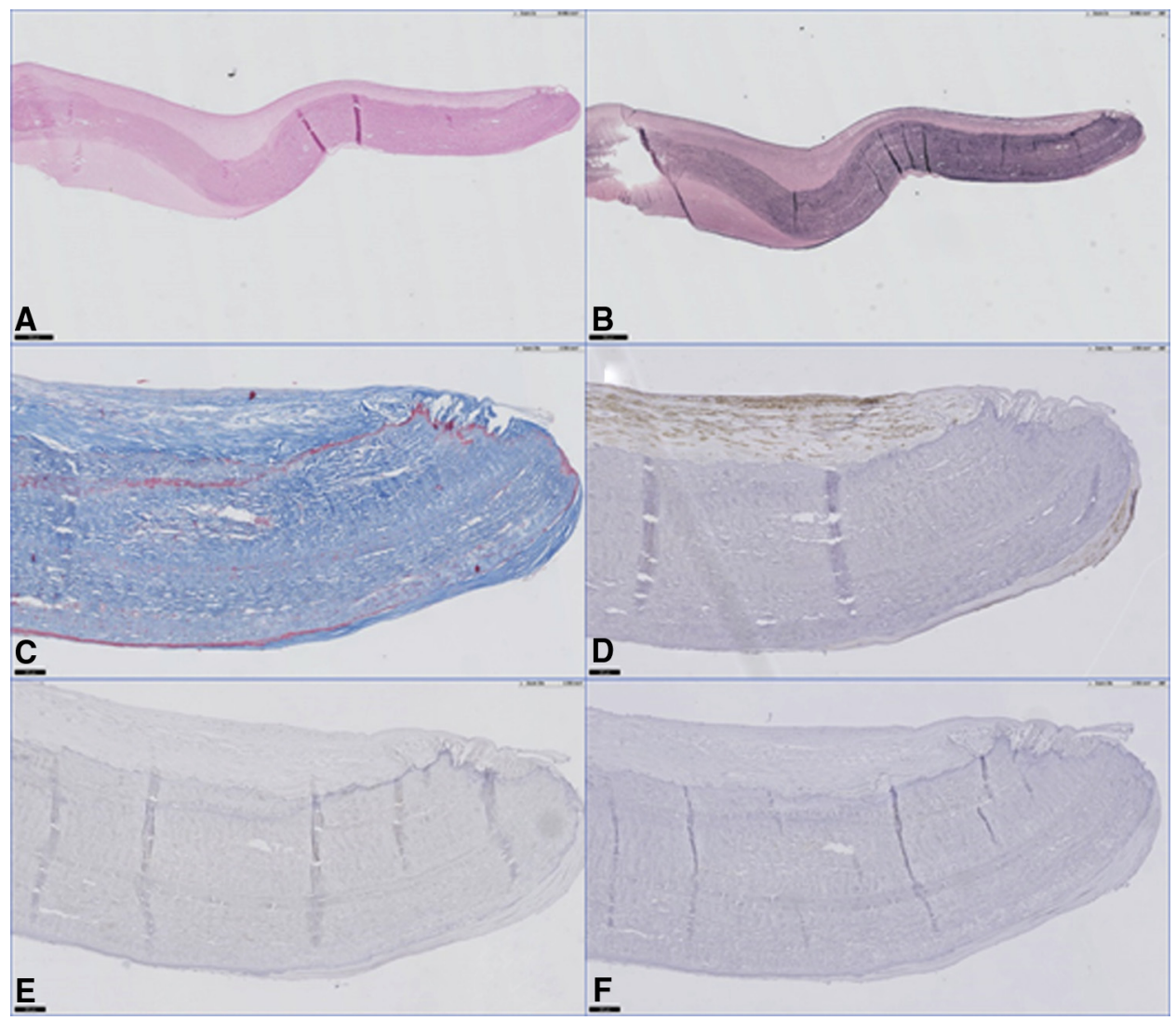

FIGURE 4. Histomorphology of implanted bovine pericardial Carpentier Edwards Perimount valves (Edwards Lifesciences, Irvine, Calif) in the pulmonary position without history of endocarditis. Collagenous thickening of the valve is seen with fibrosis. A, Hematoxylin and eosin staining, original magnification $\times 2$. B, Verhoeff-van Gieson staining, original magnification $\times 2$. C, Martius scarlet blue staining, original magnification $\times 10$. D, In the fibrosis proliferation of smooth muscle cells is depicted. Alpha smooth muscle actin staining, original magnification $\times 10$. E, No endothelial cells present. Cluster of differentiation 31 staining, original magnification $\times 10$. F, No inflammation present. Cluster of differentiation 45 staining, original magnification $\times 10$.

All explanted and examined valves showed extensive fibrotic tissue deposits. RVEDD was univariable and multivariable associated with reintervention.

\section{Reintervention}

Reintervention rates up to 10 years were acceptable in our study, with a freedom from reoperation of $95 \%$ at 5 years after surgery and $83 \%$ at 10 years postimplantation.
Beyond 10 years, intervention rates are high; however, it should be noted that only 4 patients entered that interval. Comparing reintervention rates between studies is difficult because the timing of reintervention for pulmonary valve failure is subject to intense debate and often different among congenital heart centers. ${ }^{18,19}$

Our findings show comparable or better results on freedom from reintervention at 5 and 10 years of follow-

TABLE 2. Histomorphologic characteristics of the valves that were examined

\begin{tabular}{|c|c|c|c|c|c|c|c|}
\hline Valve & $\begin{array}{c}\text { Collagenous } \\
\text { thickening }\end{array}$ & Inflammation & Calcification & Myofibroblasts & $\begin{array}{l}\text { Absence of } \\
\text { endothelium }\end{array}$ & Thrombosis/fibrin & Fibrosis \\
\hline Valve $1^{*}$ & + & Focal + & - & + & + & - & + \\
\hline Valve $2 \dagger$ & + & + & - & + & + & + & + \\
\hline Valve 3 & + & - & + & Focal + & + & + & + \\
\hline Valve 4 & + & Focal + & - & + & NA & Focal + & + \\
\hline Control valve & - & - & - & - & + & - & - \\
\hline
\end{tabular}

+, Present; Focal +, focal present; -, absent; NA, not applicable. *Underwent previous treatment for endocarditis. $\dagger$ Active endocarditis. 


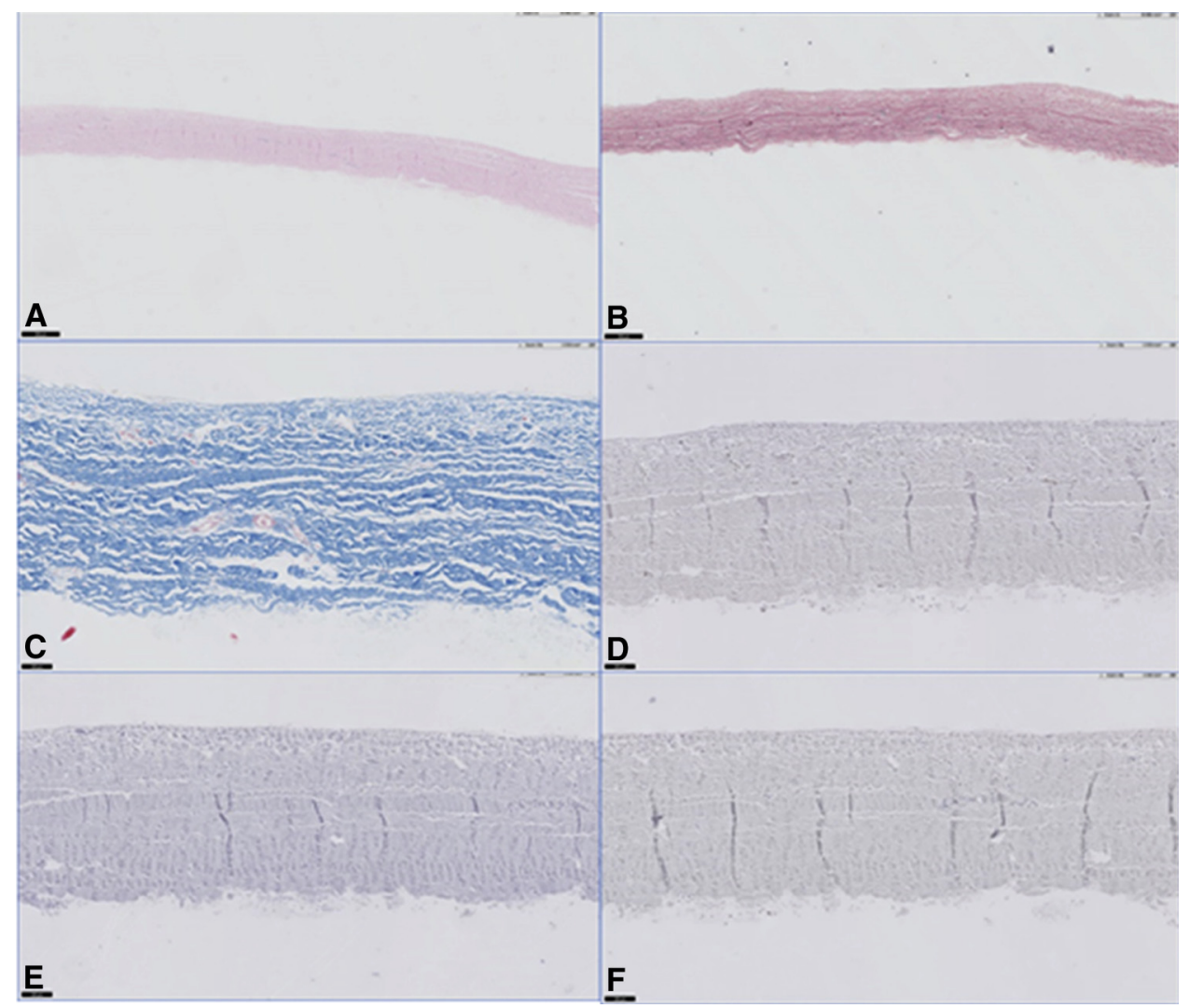

FIGURE 5. Histomorphology of normal, unimplanted bovine Carpentier Edwards Perimount valve (Edwards Lifesciences, Irvine, Calif). No calcification, fibrosis, or inflammation is present. A, Hematoxylin and eosin staining, original magnification $\times 2$. B, Verhoeff-van Gieson staining, original magnification $2 \times$. C, Martius scarlet blue staining, original magnification $\times 10$. D, Alpha smooth muscle actin staining, original magnification $\times 10$. E, Cluster of differentiation 31 staining, original magnification $\times 10$. F, Cluster of Differentiation 45 staining, original magnification $\times 10$.

up when compared with previous studies. These studies report a median freedom from reintervention of $95.2 \% \pm 15.98 \%$ (range, $50 \%-98.2 \%$ ) for 5-year followup, ${ }^{5-12,20}$ and $81.2 \%(66.4 \%-84.7 \%)$ for 10 -year followup. ${ }^{10-12}$ However, most of these studies include a younger patient population. Young age has been frequently associated with an increased risk of valve failure. ${ }^{6,11}$ When comparing with the adult population with a valve described by Nomoto and colleagues, ${ }^{12}$ freedom from reintervention was much higher in their study with $96.7 \%$ at both 5- and 10-year follow-up.

There has been a limited amount of studies reporting more than 10 years of follow-up after implantation. The study by Jang and colleagues ${ }^{11}$ reported freedom form reintervention of $21.2 \%$ at 15 years of follow-up for both pericardial and porcine valves combined. In contrast, the study by Rotes and colleagues ${ }^{10}$ had a $75.1 \%$ freedom from reintervention at 15 years for a combined cohort of $13 \%$ Perimount valves and $76 \%$ porcine valves.

In our study, we report 2 cases of successful implantation of a transcatheter valve in a failed Perimount valve. Also in literature, the option of valve-in-valve implantation in the pulmonary position seems promising. ${ }^{21,22}$ More long-term studies need to point out the reliability of this strategy.

\section{Valve Failure}

In our population we found a rather high rate of valve failure with a freedom from moderate to severe valve regurgitation of $67 \%$ at 5 years postimplantation and $59 \%$ at 10 years postimplantation. Echocardiographic evaluation of valve function has not been uniform between studies, most studies only reporting valve function at last followup. Only the study by Lee and colleagues ${ }^{19}$ presented a time related analysis. Lee and colleagues ${ }^{19}$ presented a combined end point of valve failure and dysfunction, which was $92.2 \%$ at 5 years and $20.2 \%$ at 10 years. ${ }^{20}$ Our relatively high rates of valve failure compared with the reoperation rates could be explained by the fact that a malfunctioning pulmonary valve is often tolerated quite well and timing of reintervention has been much debated. ${ }^{23}$ Also, we recognize that the choice for moderate regurgitation and moderate stenosis as definition for valve failure is arbitrarily. Choices were made as we aimed to observe the process of valve deterioration. 


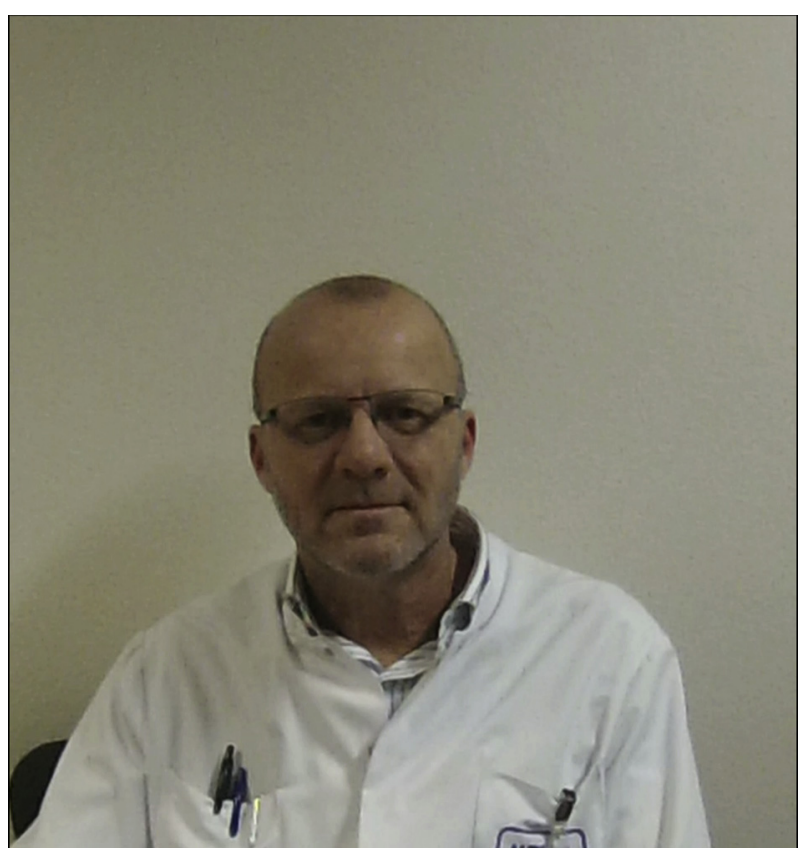

VIDEO 1. Joost van Melle, $\mathrm{MD}, \mathrm{PhD}$, explaining the importance and relevance of this study. Video available at: https://www.jtcvs.org/article/ S0022-5223(19)31355-8/fulltext.

\section{Pathology}

The explanted and histologically examined valves in our study showed stiffened leaflets that are completely retracted, likely to be caused by collagenous pannus overgrowth and the presence of myofibroblasts. To our knowledge the histology of this valve in the pulmonary position has not been studied before. Butany and colleagues $^{24}$ studied the morphology of explanted Perimount valves. However, they did not distinguish between valve locations. They showed pannus overgrowth in $69.9 \%$ of the explanted valves. ${ }^{24}$ One of their valves also showed shortening of the cusp, but this valve had been implanted in the mitral position. Jang and colleagues $^{11}$ reported macrophage infiltration, fibrotic tissue deposits, and calcium deposits on explanted pulmonary valve prosthesis; however, they did not make a distinction between the different valve types used. A recent report by Sellers and colleagues ${ }^{25}$ on transcatheter aortic valve replacement shows similarities with our findings, in a series of 23 explanted transcatheter aortic valves they showed the presence of thrombus that was followed by fibrosis. They found fibrosis on all valves implanted after 60 days, which is somewhat comparable with our results. ${ }^{25}$ Although our sample size was limited the histologic results show a distinct pattern, more structural histological evaluation is necessary to determine the failure mechanism of (pericardial) bioprosthetic valves in the pulmonary position.

\section{Limitations}

Due to the retrospective nonrandomized observational nature of this study, follow-up durations within our cohort were random. In addition, the number of patients in our study population was limited and derived from a single center. Due to the limited sample size, our models are possibly underperforming. Additional larger long-term follow-up studies are warranted to investigate this valve in the pulmonary position.

\section{CONCLUSIONS}

The Perimount valve has adequate function in the pulmonary valve position at 5 years' follow-up, after 10-year follow-up valve failure and reinterventions are common in this relatively young cohort compared with the usual tissue valve recipients (Video 1). All explanted and histologically examined valves show a similar pattern of failure showing complete retraction and stiffening of the leaflets.

\section{Conflict of Interest Statement}

Ms Pragt and Dr Ebels own rights to a patent for a mechanical valve design for the pulmonary position. Dr Mariani has been the recipient of an unrelated grant from Edwards Lifesciences. All other authors have nothing to disclose with regard to commercial support.

\section{References}

1. Pragt H, van Melle JP, Javadikasgari H, Seo DM, Stulak JM, Knez I, et al. Mechanical valves in the pulmonary position: an international retrospective analysis. J Thorac Cardiovasc Surg. 2017;154:1371-8.e1.

2. Bourguignon T, Bouquiaux-Stablo AL, Candolfi P, Mirza A, Loardi C, May MA, et al. Very long-term outcomes of the Carpentier-Edwards Perimount valve in aortic position. Ann Thorac Surg. 2015;99:831-7.

3. Bourguignon T, Bouquiaux-Stablo AL, Loardi C, Mirza A, Candolfi P, Marchand M, et al. Very late outcomes for mitral valve replacement with the Carpentier-Edwards pericardial bioprosthesis: 25-year follow-up of 450 implantations. J Thorac Cardiovasc Surg. 2014;148:2004-11.e1.

4. Johnston DR, Soltesz EG, Vakil N, Rajeswaran J, Roselli EE, Sabik JF III, et al. Long-term durability of bioprosthetic aortic valves: implications from 12,569 implants. Ann Thorac Surg. 2015;99:1239-47.

5. Chen XJ, Smith PB, Jaggers J, Lodge AJ. Bioprosthetic pulmonary valve replacement: contemporary analysis of a large, single-center series of 170 cases. $J$ Thorac Cardiovasc Surg. 2013;146:1461-6.

6. Lee C, Park CS, Lee C-H, Kwak JG, Kim SJ, Shim WS, et al. Durability of bioprosthetic valves in the pulmonary position: long-term follow-up of 181 implants in patients with congenital heart disease. J Thorac Cardiovasc Surg. 2011;142: $351-8$.

7. Kwak JG, Lee JR, Kim WH, Kim YJ. Mid-term results of the Hancock II valve and Carpentier-Edward Perimount valve in the pulmonary portion in congenital heart disease. Heart Lung Circ. 2010;19:243-6.

8. Fiore AC, Rodefeld M, Turrentine M, Vijay P, Reynolds T, Standeven J, et al. Pulmonary valve replacement: a comparison of three biological valves. Ann Thorac Surg. 2008;85:1712-8

9. Neukamm C, Lindberg HL, Try K, Dohlen G, Norgard G. Pulmonary valve replacement with a bovine pericardial valve: a five year follow-up study. World J Pediatr Congenit Heart Surg. 2014;5:534-40.

10. Rotes AS, Bonnichsen CR, Reece CL, Connolly HM, Burkhart HM, Dearani JA, et al. Long-term follow-up in repaired tetralogy of fallot: can deformation imaging help identify optimal timing of pulmonary valve replacement? J Am Soc Echocardiogr. 2014;27:1305-10. 
11. Jang W, Kim YJ, Choi K, Lim H-G, Kim W-H, Lee JR. Mid-term results of bioprosthetic pulmonary valve replacement in pulmonary regurgitation after tetralogy of Fallot repair. Eur J Cardiothorac Surg. 2012;42:e1-8.

12. Nomoto R, Sleeper LA, Borisuk MJ, Bergerson L, Pigula FA, Emani S, et al. Outcome and performance of bioprosthetic pulmonary valve replacement in patients with congenital heart disease. J Thorac Cardiovasc Surg. 2016;152:1333-42.e3.

13. Lancellotti P, Pibarot P, Chambers J, Edvardsen T, Delgado V, Dulgheru R, et al. Recommendations for the imaging assessment of prosthetic heart valves: a report from the European Association of Cardiovascular Imaging endorsed by the Chinese Society of Echocardiography, the Inter-American Society of Echocardiography, and the Brazilian Department of Cardiovascular Imaging. Eur Heart J Cardiovasc Imaging. 2016;17:589-90.

14. Baumgartner H, Hung J, Bermejo J, Chambers JB, Evangelista A, Griffin BP, et al. Echocardiographic assessment of valve stenosis: EAE/ASE recommendations for clinical practice. Eur J Echocardiogr. 2009;10:1-25.

15. Akins CW, Miller DC, Turina MI, Kouchoukos NT, Blackstone EH, Grunkemeier GL, et al. Guidelines for reporting mortality and morbidity after cardiac valve interventions. J Thorac Cardiovasc Surg. 2008;135:732-8.

16. Ascoop CAPL, van Zeijlen LGPM, Pool J, Simoins ML. Cardiac excercise testing: indications, staff, equipment, conduct and procedures. Neth J Cardiol. 1989;2:63-72.

17. Guazzi M, Adams V, Conraads V, Halle M, Mezzani A, Vanhees L, et al. Clinical recommendations for cardiopulmonary exercise testing data assessment in specific patient populations. Circulation. 2012;126:2261-74.

18. Therrien J, Provost Y, Merchant N, Williams W, Colman J, Webb G. Optimal timing for pulmonary valve replacement in adults after tetralogy of Fallot repair. Am J Cardiol. 2005;95:779-82.
19. Lee C, Jacobs JP, Lee C-H, Kwak JG, Chai PJ, Quintessenza JA. Surgical pulmonary valve insertion - when, how, and why. Cardiol Young. 2012;22: 702-7.

20. Shinkawa T, Anagnostopoulos PV, Johnson NC, Watanabe N, Sapru A, Azakie A Performance of bovine pericardial valves in the pulmonary position. Ann Thorac Surg. 2010;90:1295-300.

21. Gillespie MJ, Rome JJ, Levi DS, Williams RJ, Rhodes JF, Cheatham JP, et al. Melody valve implant within failed bioprosthetic valves in the pulmonary position: a multicenter experience. Circ Cardiovasc Interv. 2012;5: 862-70.

22. Cabalka AK, Asnes JD, Balzer DT, Cheatham JP, Gillespie MJ, Jones TK, et al. Transcatheter pulmonary valve replacement using the melody valve for treatment of dysfunctional surgical bioprostheses: a multicenter study. J Thorac Cardiovasc Surg. 2018;155:1712-24.e1.

23. Geva T. Indications for pulmonary valve replacement in repaired tetralogy of Fallot: the quest continues. Circulation. 2013;128:1855-7.

24. Butany J, Nair V, Leong SW, Soor GS, Feindel C. Carpentier-Edwards Perimount Valves? Morphological findings in surgical explants. J Card Surg. 2007; $22: 7-12$

25. Sellers S, Turner C, Sathananthan J, Cartlidge TRG, Sin F, Bouchareb R, et al. Transcatheter aortic heart valves: histological analysis providing insight to leaflet thickening and structural valve degeneration. JACC Cardiovasc Imaging. 2019; 12:135-45.

Key Words: pulmonary valve replacement, pericardial heart valves 
TABLE E1. Univariable and multivariable cox regression analyses

\begin{tabular}{|c|c|c|c|c|}
\hline \multirow[b]{2}{*}{ Variable } & \multicolumn{2}{|c|}{ Univariable } & \multicolumn{2}{|c|}{ Multivariable } \\
\hline & $\begin{array}{l}\text { Hazard ratio }(95 \% \\
\text { confidence interval) }\end{array}$ & $P$ value & $\begin{array}{l}\text { Hazard ratio }(95 \% \\
\text { confidence interval) }\end{array}$ & $P$ value \\
\hline \multicolumn{5}{|l|}{ Valve failure } \\
\hline Age & $0.97(0.92-1.02)$ & .23 & $0.98(0.93-1.03)$ & .44 \\
\hline Gender & $1.29(0.45-3.73)$ & .63 & $0.79(0.28-2.28)$ & .67 \\
\hline Sternotomies & $0.44(0.16-1.21)$ & .11 & & \\
\hline Tetralogy of Fallot & $2.05(0.71-5.93)$ & .19 & & \\
\hline Labeled size & $0.83(0.56-1.25)$ & .38 & & \\
\hline TAPSE & $1.0(0.87-1.13)$ & .98 & & \\
\hline LVEF & $1.04(0.95-1.15)$ & .38 & & \\
\hline RVEDD & $1.01(0.93-1.10)$ & .86 & & \\
\hline PPEC & $1.01(0.97-1.05)$ & .61 & & \\
\hline \multicolumn{5}{|l|}{ Reintervention } \\
\hline Age & $0.97(0.91-1.03)$ & .35 & $0.97(0.89-1.06)$ & .55 \\
\hline Gender & $0.45(0.08-2.8)$ & .40 & $0.11(0.010-1.13)$ & .063 \\
\hline Sternotomies & $0.93(0.36-2.45)$ & .89 & & \\
\hline Tetralogy of Fallot & $1.22(0.23-6.41)$ & .82 & & \\
\hline Labeled size & $0.76(0.36-1.58)$ & .50 & & \\
\hline TAPSE & $0.86(0.68-1.10)$ & .22 & & \\
\hline LVEF & $0.95(0.80-1.13)$ & .60 & & \\
\hline RVEDD & $1.24(1.01-1.51)$ & .04 & $1.39(1.04-1.85)$ & .027 \\
\hline PPEC & $1.00(0.95-1.06)$ & .94 & & \\
\hline
\end{tabular}

TAPSE, Tricuspid annular plane systolic excursion; $L V E F$, left ventricular ejection fraction; $R V E D D$, right ventricular end diastolic diameter; $P P E C$, predicted percentage exercise capacity. 